Waves and Satellites in the Near-Earth black holes, QSOs and gravitational Plasma. (Studies in Soviet Science.) By Ya L. Al'pert. Translated from Russian by Julian B. Barbour. Pp. ix +196. (Consultants Bureau: New York and London, 1974.) $\$ 42.00$.

THIS book reviews two independent phenomena observed in the magnetosphere and the solar wind: first, the way in which the plasma is disturbed when a satellite passes through it, and, second, the nature of the naturally occurring waves.

The first of three chapters outlines what is known about waves in cold and in hot magnetoplasmas: it also reviews the phenomena of particle collection, reflection, and emission from the surface of a satellite and the potential that the satellite finally attains. The second chapter reviews the phenomena that can occur in the neighbourhood of a moving satellite which may have a velocity either greater than, or comparable with, the ion thermal velocity, and a size either greater or less than the Debye length. Although there is mention of the few experiments that have been made on the topic, this chapter is largely theoretical. Chapter 3, concerned with naturally occurring waves and oscillations in the plasma, summarises the numerous experimental results that have been obtained, and gives an outline of their theoretical explanations.

This wide range of topics is discussed within the framework of a single comprehensive theory. The reader is supposed to be acquainted, at least in outline, with the details of the theory, since only the results are quoted; anyone who has that acquaintance will appreciate the skill with which the author brings out the interrelationships between the diverse phenomena.

The book has been competently translated from Russian and provides a very readable and compact account of a complicated subject. It is reasonably upto-date: of the 186 references cited most are from the past ten years, a few from the past five.

J. A. Ratcliffe

Black Holes, Gravitational Waves and Cosmology: An Introduction to Current Research. By M. Rees, R. Ruffini and J. Wheeler. Pp. 331. (Gordon and Breach: London, February 1975.) £12.80. My initial reaction on seeing this book was that, as presented, it is rather late on the scene. Though there is the making of a very useful introduction to relativistic astrophysics and cosmology here, the volume seems to have been put together out of various bits and pieces rather hurriedly without sufficient attention having been given to making the different pieces blend smoothly, or to bringing them all up to date. But that is not to say that the pieces themselves are inadequate.

Chapters 1-10 (covering chiefly pulsars, radiation) provide the largest discrete chunk, and are, according to the Preface "based on" a report by Ruffini and Wheeler which appeared in an ESRO book (SP-52) in 1971, "as updated for the present book". It would need a keen eye to spot much evidence of this updating, however, possibly because it had originally been intended to reproduce this part of the book from the ESRO publication in facsimile form. Several more recent papers are included in facsimile as appendices, but it seems a pity that the publishers have gone to the expense of resetting the first 10 chapters without obtaining substantial updating from the authors. Presumably the latter chapters,

\section{Universal phenomena}

covering cosmology, galaxy formation, and so on, represents Rees' contribution. Most of this material has suffered less from publication delays, since there have been no recent dramatic developments in cosmology, to rival the advances made in the investigation of the black hole phenomenon over the past couple of years. The attempt to make up for that deficiency by including facsimiles of a dozen or so papers published in 1970 , 1971 and 1972 is clumsy, and will not be a great help to the beginner.

I am sure that a delay in publication of another few months would have been worthwhile and would have allowed time to produce a thoroughly modernised book. The three authors are all closely involved in the work they are describing and even in the present packaging their contributions provide a valuable volume for students of such matters, especially those new to the field. I hope, however, that the publishers will enlist the aid of one of the authors in producing a more homogeneous and up-to-date second edition.

John Gribbin

Cosmic Rays: Variations and Space Ex. ploration. By L. I. Dorman. Pp. xv+ 675. (North Holland: Amsterdam and Oxford; American Elsevier: New York, 1974.) Dfl. 215 ; $\$ 82.75$.

PROFESSOR L. I. Dorman has made a massive contribution to research on cosmic ray time variations and this first volume of a revised and enlarged version of his previous work on the subject provides another scholarly tome. The book deals with the interpretation of experimental results in respect of the terrestrial atmospheric and magnetic correcting factors which must be applied to intensity measurements, and the second volume will emphasise the physics of cosmic ray modulation in interplanetary space.
Most of the energy density in cosmic radiation is carried by particles which undergo a large reduction in their intensity as they penetrate the magnetic field of the solar wind, so an interpretation of the associated time variations is necessary in order to return to the charge composition and energy spectrum of the relativistic nuclei in interstellar space. It is only when this demodulation has been achieved that a proper discussion of the origin of cosmic rays can proceed. Much of the important work determining the nearEarth values of the energy spectra for electrons, protons and heavy nuclei as a function of time, has been performed using satellite and balloon-borne instruments since the preparation of the Russian edition of this volume, although Dorman documents the relevant work up to 1966-67. Determination of the anisotropy, solar cycle variation and Forbush or magnetic storm associated effects can, however, still only be achieved to a high degree of statistical accuracy using ground-based monitors of the secondary radiation. The first harmonic of the daily variation is only about $0.2 \%$ in amplitude and the second harmonic is rather less. Yet these harmonics are related to largescale particle streaming and particle density gradients in interplanetary space and their interpretation yields information on the complete three-dimensional transport of energetic particles throughout the solar cavity.

It is in the description of the apparatus used to make these observations, of the meteorological corrections to the magnitude of the variations and of the use of the geomagnetic field and secondary particle multiplicity to determine the directional and energy dependence of the primary time variations that this book excels. Each chapter describes the historical development of the correction and interpretatation techniques. Exhaustive references are given, together with several appendices containing tables of particle cutoff energies for arrival in the geomagnetic field, information on viewing directions of cosmic ray telescopes corrected for geomagnetic bending, and meteorological correction coefficients for changes in atmospheric pressure, temperature and layer height. In the text, details of schemes for calculating the sealevel secondary products of a primary cosmic ray collision in terms of the physics of the $\pi-\mu$ and electromagnetic cascade are given.

Dorman has written an encyclopaedic account of a research method, developed mainly in the period 1950-65, which should become the standard textbook of all who need this particular tool.

J. J. Quenby 